\title{
What does the future hold for chemotherapy with the use of lipid-based nanocarriers?
}

\author{
Carlotta Pucci $i^{* \neq} \neq 1$, Chiara Martinelli ${ }^{*, \neq, 1}$ \& Gianni Ciofani ${ }^{1}$ \\ ${ }^{1}$ Smart Bio-Interfaces Department, Istituto Italiano di Tecnologia, Viale Rinaldo Piaggio 34, 56025 Pontedera, Pisa, Italy \\ *Author for correspondence: chiara.martinelli@iit.it \\ **Author for correspondence: carlotta.pucci@iit.it \\ ${ }_{\ddagger}^{\ddagger}$ Authors contributed equally
}

'6 Lipid nanosystems are very versatile and can be modified for avoiding immune system recognition, functionalized for targeting a specific tumor cell type, and engineered for responding to external stimuli"

First draft submitted: 21 November 2019; Accepted for publication: 6 December 2019; Published online: 24 December 2019

Keywords: cancer therapy $\bullet$ chemotherapeutics $\bullet$ clinical trials $\bullet$ lipid nanocarriers $\bullet$ nanomedicine

Cancer is one of the leading causes of death worldwide and, although many advances in therapy have been attained, some issues remain to be addressed. Nowadays, the most common approach is administration of chemotherapeutic agents, which are not tumor cell specific and present several side effects both at cellular (e.g., multidrug-resistance onset) and physiological levels, causing a series of adverse symptoms in already debilitated patients. In the last two decades, thanks to nanomedicine, many nanocarriers have been designed for cancer diagnosis and treatment (i.e., theranostics) [1]. Nanomaterials display many advantages with respect to conventional therapies: they present a very small size, with a high surface-to-volume ratio; they can be functionalized with specific ligands for targeting purposes; and they are known to selectively accumulate in the tumor tissues because of the enhanced permeability and retention effect [2].

Among different kinds of nanoparticles, lipid nanocarriers can encapsulate hydrophobic/hydrophilic molecules, enhancing their stability and prolonged release. Many studies have demonstrated their efficacy in association with chemotherapeutic agents. It is worth mentioning Doxil $囚$, constituted by doxorubicin-loaded poly(ethylene glycol) (PEG)-ylated liposomes, the first nanoparticles-based drug approved by the US FDA for medical application [3]. Lipid nanosystems are very versatile and can be modified for avoiding immune system recognition, functionalized for targeting a specific tumor cell type, and engineered for responding to external stimuli [4].

Many kinds of lipid-based nanoformulations are currently under evaluation in basic research and clinical context, and some of them are already commercially available. Here, we report the most innovative and recent advances in the field of lipid nanocarriers and discuss their key advantages towards cancer therapy. A description of the current clinical trials and an overview of the already approved liposomal therapies will be provided, useful not only for research scientists but also for cancer specialists and clinicians. Getting such information will help in defining which therapeutic strategy suits best for each subcategory of patients, in the perspective of designing the most efficient and personalized treatments.

\section{Lipid nanocarriers for cancer therapy Liposomes}

Significant contributions to cancer therapy have been given by liposomes, the most diffused nanovectors produced to deliver drugs to cancer cells. Liposomes are spherical vesicles formed by the self assembly of phospholipids. These are amphiphilic macromolecules that, in aqueous environments, tend to form lipid bilayers where the hydrophobic fatty acid tails are confined within the bilayer, whereas the polar heads, consisting of a phosphate group, are oriented toward the solvent. The biocompatibility of liposomes, thanks to their structure and composition similar to cell membranes, makes them ideal candidates for application in nanomedicine [2]. Their peculiar morphology allows 
them to encapsulate both hydrophilic and hydrophobic drugs. The first ones are usually enclosed in the aqueous core. Hydrophobic drugs, on the other hand, are intercalated in the lipid bilayer, limiting possible toxicity to healthy tissues and increasing their bioavailability [5]. In order to give the desired properties to liposomes, other lipids and/or cholesterol are often intercalated into the bilayer during the formulation. For instance, cholesterol is used to modulate the fluidity of the lipid bilayer in order to increase the permeability of hydrophobic drugs and stability in bodily fluids [6].

Moreover, a layer of PEG is usually added to impart steric stabilization, to extend blood circulation and to reduce uptake from mononuclear phagocytic system. Some formulations have been designed to be responsive either to external (e.g., ultrasounds, light and temperature) or internal (e.g., $\mathrm{pH}$ and specific enzymes) stimuli [7]. Knights-Mitchell \& Romanowski, as an example, developed thermosensitive liposomes loaded with doxorubicin and coated with gold, which were responsive to near-infrared laser illumination. Experiments demonstrated that the drug was released rapidly and in a more controlled way with respect to conventional chemotherapy [8].

\section{Micelles}

Micelles are spherical aggregates made of amphiphilic lipids (generally with a single lipid tail), where the lipophilic portion is packed in the nanoparticles core and the hydrophilic region faces the aqueous solvent. Hydrophobic drugs are, therefore, loaded in the core of the micelle. The hydrophilic shell, usually decorated with PEG, imparts higher solubility and stability to the system [9]. Micelles have demonstrated to be able to deliver many chemotherapeutic cargoes. Hybrid polymeric micelles, instead, are formed from the self assembly of lipids conjugated to PEG [10]. For example, PEG-phosphoethanolamine micelles were able to selectively induce apoptosis just in cancer cells, leaving healthy cells intact [11].

\section{Solid lipid nanoparticles \& nanostructured lipid carriers}

Solid lipid nanoparticles (SLNs) are composed of lipids that are solid at body temperature, such as mono, dior triglycerides, fatty acids, waxes and PEGylated lipids [2]. SLNs ensure a higher drug stability and prolonged release compared with liposomes. Moreover, the use of organic solvents is not necessary during their fabrication procedures, decreasing their toxicity profile due to the residual solvents entrapped in the particles. This also makes their preparation more biocompatible and 'green' for future scaling-up. The solid lipid matrix core allows for the encapsulation of hydrophobic drugs; however, its high crystallinity can often lead to a very low drug loading efficiency and/or very slow release kinetics.

More recently, nanostructured lipid carriers (NLCs) have been proposed in order to overcome these issues. NLCs are also composed of lipids, but at least one of them is liquid at room or body temperature. This gives rise to the formation of partially crystalline solid matrices, increasing the loading capacity of the nanocarrier [12]. This feature becomes essential for possible clinical application of these innovative nanocarriers encapsulating high amounts of drugs. Li et al. designed NLCs loaded with a combination of lapachone and doxorubicin, that were effective in overcoming multidrug resistance [13].

An interesting study demonstrated that triggered release mediated by hyperthermia was obtained by exploiting magnetic SLNs loaded with paclitaxel, allowing increased and controlled release of the drug to be obtained [14]. Recently, lipid-based magnetic nanovectors were synthesized and loaded with temozolomide. Upon stimulation with alternating magnetic fields, they released the chemotherapeutic agent in a controlled manner, inducing apoptosis of glioblastoma cells in vitro [15].

Both SLNs and NLCs have been proposed as a low-cost alternative to the other kind of lipid carriers, due to their easy preparation protocol, that can be conveniently scaled-up, and to their biocompatible and relatively cheap natural components [16].

\section{Active targeting of lipid nanocarriers}

In the attempt to reduce the side effects of chemotherapeutic drugs and to increase their efficacy, a lot of effort has been paid in making nanoparticles specific for the site of interest. Nanoparticles are known to accumulate more in tumor tissues with respect to normal ones due to their size and to different properties of tumor vasculature. This is known as the 'enhanced permeability and retention' effect. However, active targeting offers a more precise and elegant way to target only selected tissues. In order to achieve this task, the surface of the nanoparticles is functionalized with ligands that specifically interact with receptors that are overexpressed on cancer cells. Usually, the ligands that are used for this kind of applications are antibodies, peptides and proteins, small molecules (like 
folic acid) and aptamers [2]. For example, antibodies that bind to transferrin receptor have been attached to several kinds of nanoparticles because transferrin receptor is overexpressed by many tumor cells. Cell-penetrating peptides, instead, are used to favor the crossing of the blood-brain barrier; this makes the treatment of CNS diseases, such as brain cancer, more effective since a higher amount of encapsulated drug could reach the target site [17].

Notably, liposomes have been conjugated to small peptides and loaded with chemotherapeutic agents. Zhao et al. created a liposomal system carrying paclitaxel and functionalized with Glu6-RGD peptide that efficiently targeted metastatic bone cancer, showing superior efficacy with respect to the free drug itself [18]. Varshosaz et al. functionalized NLCs with trastuzumab (Herceptin) and loaded them with docetaxel, obtaining specific uptake in HER-2-positive breast cancer cells [19].

\section{Clinical trials}

Among the different kinds of lipid nanocarriers, liposomes are the most advanced in clinical practice for the treatment of several types of cancers. In particular, as already mentioned before, liposomes encapsulating doxorubicin (Doxil) were the first nanoparticles to be ever accepted by the FDA in 1995 for cancer therapy $[2,3]$. Since then, other five liposomal formulations were approved by the FDA (DaunoXome, Myocet, Mepact ${ }^{\circledR}$, Marqibo ${ }^{\circledR}$ and Onivyde $^{\circledR} /$ MM-398). Currently, clinical trials involving the use of liposomes in cancer therapy are $1862[17,20]$, 1155 of which directly include the word 'chemotherapy' in their description. In 2018, 213 clinical trials using liposomes have been started for the treatment of cancer. Interestingly, ThermoDox ${ }^{\circledR}$, a new kind of temperatureresponsive liposomal formulation, is under clinical evaluation.

Concerning micelles, basically all the ongoing clinical trials involve polymeric micelles, while none concerns the use of lipid-based micelles. Polymeric micelles are spherical aggregates formed by block co-polymers, in which the hydrophobic part forms the core of the particles whereas the hydrophilic portion constitutes the outer shell [2]. The same applies to SLNs and NLCs. The only lipid nanoparticles-based system that is currently under investigation to treat patients with refractory locally advanced or metastatic solid tumor malignancies, multiple myeloma or lymphoma is DCR-MYC, a lipid nanoparticle encapsulating a siRNA oligonucleotide targeting the proto-oncogene $M Y C$ [21]. Strictly speaking, this nanocarrier is not applied for chemotherapy; however, it represents a powerful tool in the fight against cancer. It is worth mentioning that, in parallel with chemotherapy, other kinds of approach are being studied, giving extremely interesting results. For instance, stable nucleic acid lipid particles and lipoplexes are an evolution of cationic liposomes for gene delivery purposes [22]. As an example, Scognamiglio et al. developed a transferrin-conjugated SNALP system carrying 2'-O-methylated miR-34a for multiple myeloma treatment. Mice treated with these nanomaterials displayed a significant tumor growth inhibition and increased survival rates [23]. Liposomes conjugated with chlorotoxin, a peptide specific for tumor cells and encapsulating antisense oligonucleotides or siRNAs), demonstrated to be efficiently internalized in glioma cells [24]. Gene therapy can be applied in cancer therapy by inducing the expression of proapoptotic and chemo-sensitizing genes, the expression of wild-type tumor suppressor genes or of genes able to solicit antitumor immune responses, or to deliver siRNAs for targeted gene silencing [17].

\section{Conclusion}

Reported evidences show how incredibly long and complicated is the road to the clinical practice. In fact, although a lot of nanoparticles are being studied at the research level producing an incredible amount of significant data, just few of them reach the clinical trials, and even less are finally accepted and used in clinical practice [17]. Among the main limitations for entering the clinical trial phase, it is possible to highlight: the need for a straightforward and reproducible fabrication procedure at large scale; a well-known and characterized physicochemical behavior in vitro and, possibly, in vivo; the nontoxicity of the nanoparticles; a good biodistribution and drug release kinetics. These are just few of the requisites that a lipid-based nanocarrier, or in general any kind of nanocarrier, should possess before clinical applications can be considered.

Being SLNs and NLCs fairly more recent compared with other kinds of systems, in particular compared with liposomes, their introduction in clinical trials appears to be still in the future [16]. However, given their attractive advantages, the authors hope for a rapid (and not so far in time) development in this sense. More efforts should be focused on studying the stability in vivo of these innovative systems and on finding more biocompatible and scalable formulation protocols. Finally, more representative disease animal models should be considered in order to make clinical translation step more feasible. 


\section{Open access}

This work is licensed under theAttribution-NonCommercial-NoDerivatives 4.0 Unported License. To view a copy of this license, visit http://creativecommons.org/licenses/by-nc-nd/4.0/

\section{Financial \& competing interests disclosure}

This work has received funding from the European Research Council under the EU's Horizon 2020 research and innovation program (grant agreement $N^{\circ}$ 709613, SlaMM). The authors have no other relevant affiliations or financial involvement with any organization or entity with a financial interest in or financial conflict with the subject matter or materials discussed in the manuscript apart from those disclosed.

No writing assistance was utilized in the production of this manuscript.

\section{References}

1. Shi J, Kantoff PW, Wooster R, Farokhzad OC. Cancer nanomedicine: progress, challenges and opportunities. Nat. Rev. Cancer 17(1), 20-37 (2017).

2. Martinelli C, Pucci C, Ciofani G. Nanostructured carriers as innovative tools for cancer diagnosis and therapy. APL Bioeng. 3(1), 011502 (2019).

3. Barenholz Y. Doxil ${ }^{\circledR}$ - the first FDA-approved nano-drug: lessons learned. J. Control. Rel. 160(2), 117-134 (2012).

4. Srinivasan M, Rajabi M, Mousa S. Multifunctional nanomaterials and their applications in drug delivery and cancer therapy. Nanomaterials 5(4), 1690-1703 (2015).

5. Gulati M, Grover M, Singh S, Singh M. Lipophilic drug derivatives in liposomes. Int. J. Pharm. 165(2), 129-168 (1998).

6. García-Pinel B, Porras-Alcalá C, Ortega-Rodríguez A et al. Lipid-based nanoparticles: application and recent advances in cancer treatment. Nanomaterials 9(4), 638 (2019).

7. Zangabad PS, Mirkiani S, Shahsavari S et al. Stimulus-responsive liposomes as smart nanoplatforms for drug delivery applications. Nanotechnol. Rev. 7(1), 95-122 (2018).

8. Knights-Mitchell SS, Romanowski M. Near-infrared activated release of doxorubicin from plasmon resonant liposomes. Nanotheranostics 2(4), 295-305 (2018).

9. Salmaso S, Caliceti P. Stealth properties to improve therapeutic efficacy of drug nanocarriers. J. Drug Deliv. 2013, 374252 (2013).

10. Hanafi NAN, El-Kemary M, Leporatti S. Micelles structure development as a strategy to improve smart cancer therapy. Cancers (Basel) 10(7), pii: E238 (2018).

11. Wang J, Fang X, Liang W. Pegylated phospholipid micelles induce endoplasmic reticulum-dependent apoptosis of cancer cells but not normal cells. ACS Nano 6(6), 5018-5030 (2012).

12. Tamjidi F, Shahedi M, Varshosaz J, Nasirpour A. Nanostructured lipid carriers (NLC): a potential delivery system for bioactive food molecules. Innov. Food Sci. Emerg. Technol. 19, 29-43 (2013).

13. Li X, Jia X, Niu H. Nanostructured lipid carriers co-delivering lapachone and doxorubicin for overcoming multidrug resistance in breast cancer therapy. Int. J. Nanomedicine 13, 4107-4119 (2018).

14. Oliveira RR, Carrião MS, Pacheco MT et al. Triggered release of paclitaxel from magnetic solid lipid nanoparticles by magnetic hyperthermia. Mater. Sci. Eng. C 92, 547-553 (2018).

15. Tapeinos C, Marino A, Battaglini M et al. Stimuli-responsive lipid-based magnetic nanovectors increase apoptosis in glioblastoma cells through synergic intracellular hyperthermia and chemotherapy. Nanoscale 11(1), 72-88 (2018).

16. Puri A, Loomis K, Smith B et al. Lipid-based nanoparticles as pharmaceutical drug carriers: from concepts to clinic. Crit. Rev. Ther. Drug Carrier Syst. 26(6), 523-580 (2009).

17. Pucci C, Martinelli C, Ciofani G. Innovative approaches for cancer treatment: current perspectives and new challenges. Ecancermedicalscience 13, 961 (2019).

18. Zhao Z, Zhao Y, Xie C et al. Dual-active targeting liposomes drug delivery system for bone metastatic breast cancer: synthesis and biological evaluation. Chem. Phys. Lipids 223, 104785 (2019).

19. Varshosaz J, Davoudi MA, Rasoul-Amini S. Docetaxel-loaded nanostructured lipid carriers functionalized with trastuzumab (Herceptin) for HER2-positive breast cancer cells. J. Liposome Res. 28(4), 285-295 (2018).

20. Clinical Trials Database:NCT00193037 (2013). https://clinicaltrials.gov/ct2/show/NCT00193037?term=NCT00193037\&draw=2\&rank=1

21. Clinical Trials Database:NCT02110563 (2017). https://clinicaltrials.gov/ct2/show/NCT021 10563?term=solid+lipid+nanoparticles\&draw=2\&rank=3

22. Campani V, Salzano G, Lusa S, De Rosa G. Lipid nanovectors to deliver RNA oligonucleotides in cancer. Nanomaterials 6(7), pii E131 (2016). 
23. Scognamiglio I, Di Martino MT, Campani V et al. Transferrin-conjugated SNALPs encapsulating 2'-O-methylated miR-34a for the treatment of multiple myeloma. Biomed. Res. Int. 2014, 217365 (2014).

24. Costa PM, Cardoso AL, Mendonça LS et al. Tumor-targeted chlorotoxin-coupled nanoparticles for nucleic acid delivery to glioblastoma cells: a promising system for glioblastoma treatment. Mol. Ther. Nucleic Acids 2, e100 (2013). 
